\title{
Glycaemic variability is associated with microvascular complications in Emiratis with Type 2, but not Type I diabetes
}

\author{
Mohamed Suliman', Adam Buckley², Nader Lessan² and Maha T Barakat ${ }^{2}$ \\ ' Imperial College London Diabetes Centre, (Al Ain, United Arab Emirates) \\ ${ }^{2}$ Imperial College London Diabetes Centre, (Abu Dhabi, United Arab Emirates)
}

\section{Background}

Microvascular complications of diabetes mellitus are a major source of morbidity. Variability in blood glucose may contribute to the risk of these complications.

\section{Aims}

To determine the contribution of $\mathrm{HbAlc}$ or early morning serum glucose contribute to the risk of microvascular complications, controlling for established risk factors.

\section{Methods}

ICLDC patient database was accessed to identify people of Emirati origin with type I $(n=994)$ or type $2(n=18,904)$ diabetes, who underwent retinal screening between $01 / 01 / 2015$ and 01/04/2017, with at least five recorded measurements of $\mathrm{HbAl} \mathrm{c}$ and plasma glucose.

"Average" and "variability" of each were expressed as the mean and standard deviation (SD).

Retinal disease in the more severely affected eye was classified as: "none"

(ROMO), "background" (RIMO) and "significant" (any higher grade).

Microalbuminuria was used as the indicator of nephropathy and was defined as urinary albumin/creatinine ratio $\geq 2.5 \mathrm{mg} / \mathrm{mmol}$ in females or $\geq 3.5 \mathrm{mg} / \mathrm{mmol}$ in males at both of the two most recent measurements.

Smoking was graded in ordered fashion as: "never smoked", "passive smoking", "ex-smoker" and "current smoker"

Logistic regression models were used to examine the contribution of average and variability of $\mathrm{HbAlc}$ and glucose to the likelihood of retinal or renal endpoints, controlling for diabetes duration, smoking, and most recently measured systolic blood pressure (sBP). Interaction terms were included for means and SDs of both $\mathrm{HbAlc}$ and glucose.

\section{Results}

Patients with type I diabetes had higher mean HbAIc ( $8.8 \%$ cf $7.4 \%, p<0.000$ I), mean glucose ( $11.7 \mathrm{mmol} / \mathrm{L}$ cf $9.1 \mathrm{mmol} / \mathrm{L}, p<0.000 \mathrm{I}$ ), SD HbAlc ( $1.18 \%$ cf $0.88 \%, p<0.000 \mathrm{I}$ ), and SD glucose $(5.12 \mathrm{mmol} / \mathrm{L}$ cf $2.25 \mathrm{mmol} / \mathrm{L}, \mathrm{p}<0.000 \mathrm{I})$ compared to those with type 2 diabetes.
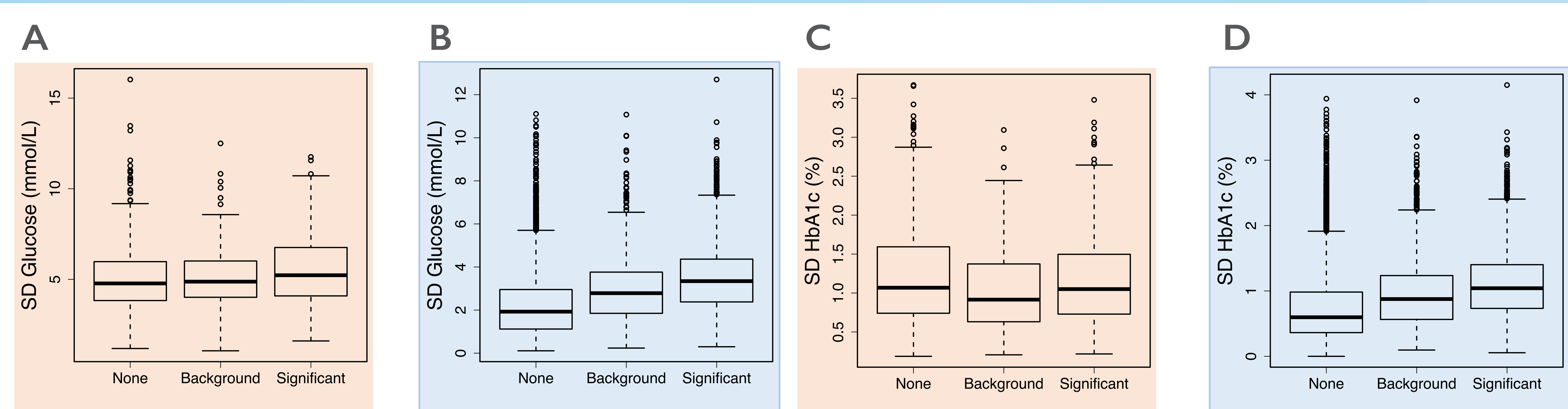

Figures: Standard deviation (SD) of morning glucose ( $\mathrm{mmol} / \mathrm{L})$ by grade of retinal disease in people with Type I (A) and Type 2 (B) diabetes. SD of HbAlc (\%) by grade of retinal disease in people with Type I (C) and type 2 (D) diabetes.

In both type I and type 2 diabetes, independent and significant predictors of microvascular complications (retinopathy and nephropathy) included mean HbAlc and diabetes duration; in addition, systolic blood pressure and smoking were independent predictors of nephropathy.

SD HbAIc and SD Glucose were independent predictors of microvascular complications (retinopathy and nephropathy) in type 2 (table I), but NOT type I diabetes.

In type 2 (but NOT type I) diabetes, the interaction between SD and mean HbA Ic was significant for nephropathy and background retinopathy end points, indicating the effect of $\mathrm{HbAl}$ c was greatest at lower values of mean $\mathrm{HbAI}$; the interaction between SD and mean glucose was significant for nephropathy and background retinopathy endpoints.

\begin{tabular}{l}
\hline \multicolumn{9}{c|}{ Type 2 } \\
\hline
\end{tabular}

Acknowledgements

This study was supported by Imperial College London Diabetes Centre

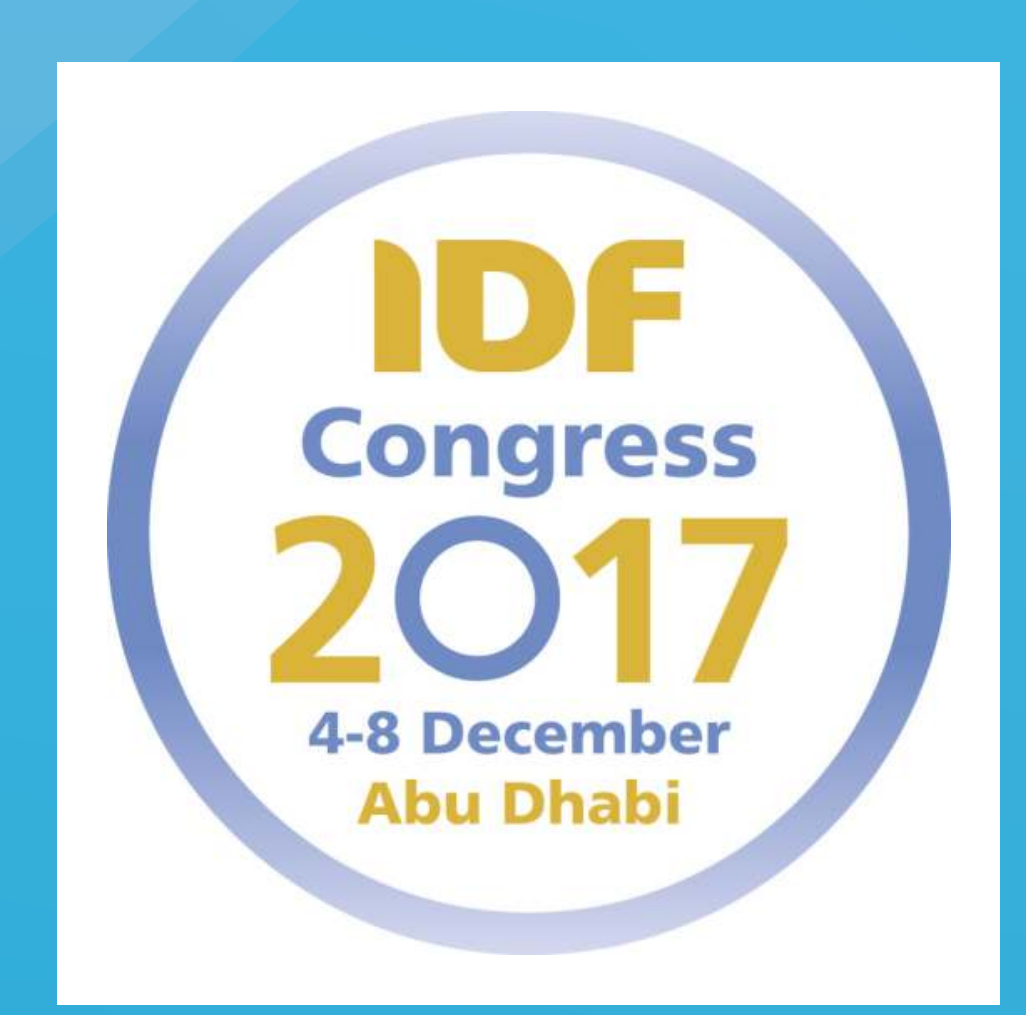

\section{Discussion}

Glycaemic variability is an independent predictor of microvascular complications in type 2 diabetes, but its predictive power diminishes with increasing average $\mathrm{HbAlc}$ and glucose.

Neither variability of glucose nor HbAlc significantly predicted microvascular outcomes in Emirati people with type I diabetes, despite higher variability of both than found in people with type 2 diabetes. 